\title{
Implications of the B-mode Polarization Measurement for Direct Detection of Inflationary Gravitational Waves
}

\author{
Sachiko Kuroyanagi, ${ }^{1}$ Shinji Tsujikawa, ${ }^{1}$ Takeshi Chiba, ${ }^{2}$ and Naoshi Sugiyama ${ }^{3,4}$ \\ ${ }^{1}$ Department of Physics, Faculty of Science, Tokyo University of Science, \\ 1-3, Kagurazaka, Shinjuku-ku, Tokyo 162-8601, Japan \\ ${ }^{2}$ Department of Physics, College of Humanities and Sciences, Nihon University, Tokyo 156-8550, Japan \\ ${ }^{3}$ Department of Physics, Nagoya University, Chikusa, Nagoya 464-8602, Japan \\ ${ }^{4}$ Kavli Institute for Physics and Mathematics of the Universe, University of Tokyo, Chiba 277-8582, Japan
}

(Dated: September 12, 2018)

\begin{abstract}
The prospects for direct measurements of inflationary gravitational waves by next generation interferometric detectors inferred from the possible detection of B-mode polarization of the cosmic microwave background are studied. We compute the spectra of the gravitational wave background and the signal-to-noise ratios by various types of interferometric detectors (DECIGO, BBO, and Ultimate DECIGO) for large-field inflationary models in which the tensor-to-scalar ratio is greater than the order of 0.01 . If the reheating temperature $T_{\mathrm{RH}}$ of chaotic inflation with the quadratic potential is high $\left(T_{\mathrm{RH}}>7.9 \times 10^{6} \mathrm{GeV}\right.$ for upgraded DECIGO, $T_{\mathrm{RH}}>1.8 \times 10^{6} \mathrm{GeV}$ for $\mathrm{BBO}$, and $T_{\mathrm{RH}}>2.8 \times 10^{3} \mathrm{GeV}$ for Ultimate DECIGO), it will be possible to reach the sensitivity of the gravitational background in future experiments at $3 \sigma$ confidence level. The direct detection is also possible for natural inflation with the potential $V(\phi)=\Lambda^{4}[1-\cos (\phi / f)]$, provided that $f>4.2 M_{\mathrm{pl}}$ (upgraded DECIGO) and $f>3.6 M_{\mathrm{pl}}(\mathrm{BBO})$ with $T_{\mathrm{RH}}$ higher than $10^{8} \mathrm{GeV}$. The quartic potential $V(\phi)=\lambda \phi^{4} / 4$ with a non-minimal coupling $\xi$ between the inflaton field $\phi$ and the Ricci scalar $R$ gives rise to a detectable level of gravitational waves for $|\xi|$ smaller than the order of 0.01 , irrespective of the reheating temperature.
\end{abstract}

PACS numbers: $98.80 . \mathrm{Cq}, 95.30 . \mathrm{Cq}$

\section{INTRODUCTION}

The inflationary paradigm was originally proposed to address the horizon, flatness, and monopole problems in the standard big-bang cosmology [1]. Moreover, inflation can be responsible for the generation of primordial density perturbations [2] and gravitational waves [3] by stretching quantum fluctuations over super-Hubble scales. The simplest slow-roll inflationary scenario driven by the potential energy of a single scalar field gives rise to nearly scale-invariant primordial power spectra of scalar and tensor perturbations.

While the primordial scalar perturbation has been observed by COBE [4], WMAP [5], and Planck [6] satellites as a form of temperature anisotropies of the Comic Microwave Background (CMB), gravitational waves had eluded the detection for a long time. This is attributed to the fact that the relative amplitude of gravitational waves to scalar perturbations is suppressed in the standard inflationary scenario (see Refs. 7] for reviews).

The tensor-to-scalar ratio $r$ and the spectral index $n_{s}$ of scalar perturbations are two important quantities to probe models of inflation by using CMB observations. Although the Planck satellite measured $n_{s}$ in high precision, this is not sufficient to narrow down the allowed models of inflation unless $r$ is constrained tightly [ 8 [10]. The detection of primordial gravitational waves is crucial to break the degeneracy between inflationary models. In fact, there is the consistency relation between $r$ and the spectral index of primordial gravitational waves $n_{t}: r=-8 n_{t}$ for potentialdriven slow-roll inflation [11]. The detection of gravitational waves is a litmus test for inflationary cosmology.

Recently, the BICEP2 collaboration reported the evidence for the detection of B-mode polarization in the CMB [12]. They claimed that the observed B-mode can be fit by a lensed $\Lambda$-Cold-Dark-Matter $(\Lambda \mathrm{CDM})$ model with $r=0.2_{-0.05}^{+0.07}$. This bound is larger than the upper limit $r<0.11$ derived by the Planck data of temperature anisotropies [6], $[\underline{8}]$ combined with the WMAP large-angle polarization (WP) data 13]. There is also an argument that the presence of the polarized dust weakens the constraints on $r$ 14]. Since the BICEP2 measured the B-mode polarization at a single frequency $(150 \mathrm{GHz})$, the upcoming independent observations at different frequencies with precise measurements of the dust polarization will be crucial to clarify whether the signal is really the cosmological origin or not.

If the B-mode polarization reported by BICEP2 comes from the primordial origin, it marks a milestone in the inflationary cosmology [15]. Since the primordial B-mode can be generated only from tensor or vector perturbations [16] and vector perturbations sourced by topological defects are disfavored by the CMB data [17], the detection of B-mode polarization indicates an indirect discovery of inflationary gravitational waves. A relatively large value of the tensor-to-scalar ratio of the order of 0.1 can be explained by the so-called large field inflationary models in which the field variation $\Delta \phi$ during inflation is greater than $10 M_{\mathrm{pl}}$, where $M_{\mathrm{pl}}=2.435 \times 10^{18} \mathrm{GeV}$ is the reduced Planck mass [18]. 
The typical examples of the large-field models are chaotic inflation [19] and natural inflation [20]. Although chaotic inflation with quartic potential is disfavored by the Planck data [8], the non-minimal coupling [21 23] can reduce the value of $r$ [24, 25] so that the model can be compatible with the data. A similar property also holds in the presence of Galileon couplings [26] and field derivative couplings to the Einstein tensor [27].

If the large-field models correspond to a realistic paradigm of inflation, the next generation interferometric detectors such as DECIGO [28] and BBO [29] may allow us to detect inflationary gravitational waves directly. These instruments are designed to measure tensor perturbations at much shorter wavelengths relative to the perturbations associated with the CMB B-mode polarization. These frequencies correspond to the mode that re-entered the Hubble radius during the radiation-dominated epoch. The direct detection of gravitational waves 30 36 contains useful information of the very early Universe, such as the effective relativistic degrees of freedom [37, 38] and the reheating temperature after inflation [39, 40].

In this paper, we compute the spectra of the gravitational wave background for several large-field inflationary models: chaotic inflation, natural inflation and non-minimally coupled inflation with quartic potential. Then, we update the previous calculations [41] of signal-to-noise ratios by DECIGO and BBO with various reheating temperatures. We also calculate signal-to-noise ratio for Ultimate DECIGO whose sensitivity is only limited by quantum noise [28]. We show that, even if these models are degenerate in terms of the CMB observables $n_{s}$ and $r$, it is possible to distinguish them from the direct detection of inflationary gravitational waves.

This paper is organized as follows. In Sec. II we present background equations of motion during inflation/reheating in the presence of non-minimal couplings. In Sec. III we review primordial gravitational waves generated during slow-roll inflation including non-minimal couplings between the inflaton field $\phi$ and the Ricci scalar $R$. In Sec. IV] we calculate the spectra of the gravitational background for the three large-field inflationary models by using the bounds derived from the recent CMB data. In Sec. V] we study the detectability of inflationary gravitational waves by computing the signal-to-noise ratio (SNR) associated with upgraded DECIGO, BBO, and Ultimate DECIGO. Section VI is devoted to summary.

\section{BACKGROUND EQUATIONS OF MOTION DURING INFLATION AND REHEATING}

We start with the inflationary model given by the action

$$
S=\int d^{4} x \sqrt{-g}\left[\frac{M_{\mathrm{pl}}^{2}}{2} R-\frac{1}{2} g^{\mu \nu} \partial_{\mu} \phi \partial_{\nu} \phi-V(\phi)-\frac{1}{2} \xi \phi^{2} R\right]
$$

where $R$ is the Ricci scalar of the metric $g_{\mu \nu}, V(\phi)$ is the potential of a scalar field $\phi$, and $\xi$ is the non-minimal coupling. In our sign convention, the conformal coupling corresponds to $\xi=1 / 6$. We study the following three models in which the variation of the field during inflation is greater than the order of $M_{\mathrm{pl}}$ :

$$
\begin{aligned}
\text { (i) } V & =\frac{1}{2} m^{2} \phi^{2}, \quad \xi=0, \\
\text { (ii) } V & =\Lambda^{4}\left[1-\cos \left(\frac{\phi}{f}\right)\right], \quad \xi=0, \\
\text { (iii) } V & =\frac{1}{4} \lambda \phi^{4}, \quad \xi \neq 0,
\end{aligned}
$$

where $m, \Lambda, f$, and $\lambda$ are constants. The models (i) and (iii) belong to the class of chaotic inflation [19] with the quadratic potential and the quartic potential, respectively. In the case (iii), we have introduced the non-minimal coupling $\xi$, as this can reduce the tensor-to-scalar ratio $r$. The potential of the model (ii) corresponds to that of natural inflation [20]. The slow-roll inflation is possible for $f>M_{\mathrm{pl}}$. For large decay constant $f \gg M_{\mathrm{pl}}$, it is known that natural inflation is indistinguishable from quadratic chaotic inflation.

\section{A. Background equations of motion}

We consider the flat Friedmann-Lemaître-Robertson-Walker (FLRW) background described by the line-element $d s^{2}=-d t^{2}+a^{2}(t) \delta_{i j} d x^{i} d x^{j}$, where $a(t)$ is the scale factor with cosmic time $t$. During the reheating after inflation, the inflaton energy density decays into the radiation energy density $\rho_{r}$. The Born decay to light particles (decay constant $\Gamma$ ) during the oscillating stage of a scalar field can be effectively described by taking into account the friction 
term $\Gamma \dot{\phi}$ to the inflaton equation of motion [42, 43] (a dot represents a derivative with respect to $t$ ). Then, the Friedmann equation and the scalar field equation of motion are given, respectively, by

$$
\begin{aligned}
& 3 H^{2}\left(M_{\mathrm{pl}}^{2}-\xi \phi^{2}\right)=\frac{1}{2} \dot{\phi}^{2}+V(\phi)+6 H \xi \phi \dot{\phi}+\rho_{r}, \\
& \ddot{\phi}+(3 H+\Gamma) \dot{\phi}+V_{, \phi}+6\left(2 H^{2}+\dot{H}\right) \xi \phi=0,
\end{aligned}
$$

where $V_{, \phi} \equiv d V / d \phi$ and $H \equiv \dot{a} / a$ is the Hubble parameter. We caution that Eq. (6) with the $\Gamma \dot{\phi}$ term is valid only during the oscillating stage of the inflaton around the potential minimum [44]. Provided that $H \gg \Gamma$ during inflation, the decay term $\Gamma \dot{\phi}$ does not play any significant role until the onset of reheating.

Due to the energy conservation, the radiation density $\rho_{r}$ obeys the equation of motion

$$
\dot{\rho}_{r}+4 H \rho_{r}=\Gamma \dot{\phi}^{2}
$$

From Eq. (5), we obtain

$$
H=\frac{6 \xi \phi \dot{\phi}+\sqrt{6\left[2\left(V+\rho_{r}\right)\left(M_{\mathrm{pl}}^{2}-\xi \phi^{2}\right)+\dot{\phi}^{2}\left\{M_{\mathrm{pl}}^{2}+\xi \phi^{2}(6 \xi-1)\right\}\right]}}{6\left(M_{\mathrm{pl}}^{2}-\xi \phi^{2}\right)}
$$

Taking the time derivative of Eq. (5) and eliminating the term $\dot{H}$ on account of Eq. (6), we have

$$
\begin{aligned}
\ddot{\phi}= & -\left\{M_{\mathrm{pl}}^{2}\left[(3 H+\Gamma) \dot{\phi}+V_{, \phi}\right]-\phi\left[3 \dot{\phi}^{2}-12 H^{2} M_{\mathrm{pl}}^{2}+(3 H+\Gamma) \phi \dot{\phi}+\phi V_{, \phi}+4 \rho_{r}\right] \xi\right. \\
& \left.+6 \phi(\dot{\phi}-2 H \phi)(\dot{\phi}+H \phi) \xi^{2}\right\} /\left[M_{\mathrm{pl}}^{2}+\xi \phi^{2}(6 \xi-1)\right] .
\end{aligned}
$$

We solve the differential equations (7) and (9) with Eq. (8).

For the models (i) and (ii), the slow-roll inflationary stage is followed by oscillations of the massive inflaton field. This corresponds to the temporal matter era during which the evolution of the scale factor is given by $a \propto t^{2 / 3}$. Around the time $t_{\mathrm{RH}} \simeq \Gamma^{-1}, \rho_{r}$ catches up with the inflaton energy density $\rho_{\phi}=\dot{\phi}^{2} / 2+V(\phi)$ [45]. Here, the subscript "RH" denotes the value at the end of reheating. We numerically find that the radiation energy density at $t=t_{\mathrm{RH}}$ is given by $\rho_{r}\left(t_{\mathrm{RH}}\right) \simeq 0.5 \Gamma^{2} M_{\mathrm{pl}}^{2}$. On using the relation $\rho_{r}(T)=\left(\pi^{2} / 30\right) g_{*}(T) T^{4}$, where $T$ is the temperature and $g_{*}$ is the number of relativistic degree of freedom, the reheating temperature is given by

$$
T_{\mathrm{RH}} \simeq 1.1 g_{*, \mathrm{RH}}^{-1 / 4}\left(\Gamma M_{\mathrm{pl}}\right)^{1 / 2}
$$

We take $g_{*, \mathrm{RH}}=106.75$ for the value of $g_{*}$ at the end of reheating. For $t>t_{\mathrm{RH}}$, the Universe enters the radiationdominated epoch.

\section{B. Dynamics of reheating for the model (iii) with $|\xi| \ll 1$}

In the model (iii), the CMB observables $n_{s}$ and $r$ are subject to change relative to the case $\xi=0$ because the effect of non-minimal couplings cannot be neglected due to the large inflaton value. As we will see in Sec. IIIC, the model is compatible with the observational data even for $|\xi| \ll 1$. Since the amplitude of the field $\phi$ drops below $M_{\mathrm{pl}}$ after inflation, the effect of non-minimal couplings on the background Eqs. (77)- (9) should be negligible during reheating for $|\xi| \ll 1$. In this case, the dynamics of reheating is driven by a massless inflaton field, so that the scale factor evolves as $a \propto t^{1 / 2}$ and hence, there is no transient matter era between inflation and the radiation-dominated epoch.

For the model (iii) with $|\xi| \ll 1$, the dynamics of reheating can be analytically known by using the virial theorem $\left\langle\dot{\phi}^{2} / 2\right\rangle=2\langle V(\phi)\rangle$, where $\langle\cdots\rangle$ corresponds to the time average over inflaton oscillations. Dropping the contribution of non-minimal couplings in Eq. (6), it follows that

$$
\left\langle\dot{\rho}_{\phi}\right\rangle+(4 H+4 \Gamma / 3)\left\langle\rho_{\phi}\right\rangle \simeq 0
$$

Integration of Eq. (11) gives

$$
\left\langle\rho_{\phi}\right\rangle=\rho_{\phi i}\left(\frac{t_{i}}{t}\right)^{2} e^{-(4 / 3) \Gamma\left(t-t_{i}\right)}
$$


where the subscript " $i$ " is used for quantities at the onset of inflaton oscillations and we used the solution $a=a_{i}\left(t / t_{i}\right)^{1 / 2}$ for $t>t_{i}$. Substituting Eq. (12) into Eq. (77), i.e., $\left\langle\dot{\rho}_{r}\right\rangle+4 H\left\langle\rho_{r}\right\rangle=(4 / 3) \Gamma\left\langle\rho_{\phi}\right\rangle$, we obtain the following solution

$$
\left\langle\rho_{r}\right\rangle=\rho_{\phi i}\left(\frac{t_{i}}{t}\right)^{2}\left[1-e^{-(4 / 3) \Gamma\left(t-t_{i}\right)}\right] .
$$

The time $t_{i}$ can be estimated as $t_{i} \simeq 1 /\left(2 H_{i}\right)$. Provided that $\Gamma \ll H_{i}$, the time $t_{\mathrm{RH}}$ at which $\left\langle\rho_{r}\right\rangle$ equals $\left\langle\rho_{\phi}\right\rangle$ is given by $t_{\mathrm{RH}} \simeq 0.52 \Gamma^{-1}$. On using the relation $\rho_{\phi i} \simeq 3 M_{\mathrm{pl}}^{2} H_{i}^{2}$, we have $\left\langle\rho_{r}\right\rangle\left(t_{\mathrm{RH}}\right) \simeq 1.4 \Gamma^{2} M_{\mathrm{pl}}^{2}$. Equating this with the radiation density $\left(\pi^{2} / 30\right) g_{*, \mathrm{RH}} T_{\mathrm{RH}}^{4}$, it follows that

$$
T_{\mathrm{RH}} \simeq 1.4 g_{*, \mathrm{RH}}^{-1 / 4}\left(\Gamma M_{\mathrm{pl}}\right)^{1 / 2} .
$$

We have numerically solved (7) and (9) with Eq. (8) from the onset of reheating and confirmed that the above analytic estimation shows good agreement with our numerical results for $|\xi| \ll 1$.

In Sec. V we employ the relations (10) and (14) to estimate $T_{\mathrm{RH}}$ for a given decay constant $\Gamma$. As we see in Sec. IV. the absence of the temporal matter era for the model (iii) affects the resulting spectrum of the gravitational wave background relative to the models (i) and (ii).

\section{The number of e-foldings during inflation}

The number of e-foldings during inflation is defined by $N(k) \equiv \ln \left(a_{\text {end }} / a_{k}\right)$, where $a_{k}$ is the value of $a$ when a wave number $k$ equals $a H$ during inflation and $a_{\text {end }}$ is its value at the end of inflation. This quantity is related to the observables such as $n_{s}$ and $r$, so the accurate estimation of $N(k)$ is necessary to place concrete constraints on inflationary models. After inflation, there is a reheating stage followed by the radiation-dominated epoch. Then, the wave number $k=a_{k} H_{k}$ divided by $a_{0} H_{0}$ (the label " 0 " represents today's values) reads

$$
\frac{k}{a_{0} H_{0}}=e^{-N(k)} \frac{a_{\mathrm{end}}}{a_{\mathrm{RH}}} \frac{a_{\mathrm{RH}}}{a_{0}} \frac{H_{k}}{H_{0}},
$$

where we express the Hubble constant as $H_{0}=2.133 h \times 10^{-42} \mathrm{GeV}$ and $a_{0} H_{0}=2.235 \times 10^{-4}(h / 0.67) \mathrm{Mpc}^{-1}$.

We assume that the entropy at the end of reheating (with the relativistic degrees of freedom $g_{s, \mathrm{RH}}$ ) is conserved in the photon and neutrino background today. This leads to the following relation [46]):

$$
g_{s, \mathrm{RH}} T_{\mathrm{RH}}^{3} a_{\mathrm{RH}}^{3}=\left[2 T_{0}^{3}+\frac{7}{8} \cdot 6 T_{\nu 0}^{3}\right] a_{0}^{3},
$$

where $T_{0}=2.725 \mathrm{~K}=2.348 \times 10^{-13} \mathrm{GeV}$ is the CMB temperature at present and today's temperature of neutrinos is given by $T_{\nu 0}=(4 / 11)^{1 / 3} T_{0}$. Then, we obtain

$$
\frac{a_{0}}{a_{\mathrm{RH}}}=\left(\frac{11}{43} g_{s, \mathrm{RH}}\right)^{1 / 3} \frac{T_{\mathrm{RH}}}{T_{0}} .
$$

If the total energy density $\rho$ during reheating is given by $\rho \propto a^{-q}$, where $q$ is constant, the number of e-foldings in the reheating period can be estimated as $N_{\mathrm{RH}} \equiv \ln \left(a_{\mathrm{RH}} / a_{\mathrm{end}}\right)=(1 / q) \ln \left(\rho_{\mathrm{end}} / \rho_{\mathrm{RH}}\right)$. The energy density $\rho_{\mathrm{RH}}$ is related to the reheating temperature $T_{\mathrm{RH}}$, as $\rho_{\mathrm{RH}}=\left(\pi^{2} / 30\right) g_{*, \mathrm{RH}} T_{\mathrm{RH}}^{4}$, where $g_{*, \mathrm{RH}}$ is the number of relativistic degrees of freedom at the end of reheating.

On using the aforementioned relations in Eq. (15), it follows that

$$
N(k)=-\ln \left(\frac{k}{a_{0} H_{0}}\right)-\frac{1}{q} \ln \left(\frac{30}{\pi^{2}}\right)-\frac{1}{3} \ln \left(\frac{11}{43}\right)+\ln \left(\frac{g_{*, \mathrm{RH}}^{1 / q}}{g_{s, \mathrm{RH}}^{1 / 3}}\right)-\ln \left(\frac{\rho_{\mathrm{end}}^{1 / q}}{T_{\mathrm{RH}}^{4 / q-1} T_{0}}\right)+\ln \left(\frac{H_{k}}{H_{0}}\right) .
$$

Here the Hubble parameter $H_{k}$ is associated with the inflationary energy density $\rho_{\mathrm{inf}}$, as $H_{k}=\sqrt{\rho_{\mathrm{inf}} /\left(3 M_{\mathrm{pl}}^{2}\right)}$. Since $\rho_{\text {end }}$ differs from $\rho_{\text {inf }}$, we define

$$
\alpha \equiv \frac{\rho_{\text {end }}}{\rho_{\text {inf }}},
$$

to quantify their difference. In the following, we set $g_{*, \mathrm{RH}}=g_{s, \mathrm{RH}}$ by assuming that no entropy production occurs after the reheating stage until the neutrino decoupling $(\sim \mathrm{MeV})$. Depending on the values of $q$, we shall discuss two qualitatively different cases. 


\section{Massive inflaton $(q=3)$}

The models (i) and (ii) belong to the class in which the energy density during reheating decreases as $\rho \propto a^{-3}$. In this case, the number of e-foldings (18) reads

$$
N(k)=55.9-\frac{1}{3} \ln \alpha-\ln \left(\frac{k}{a_{0} H_{0}}\right)-\ln \left(\frac{h}{0.67}\right)+\frac{1}{3} \ln \left(\frac{T_{\mathrm{RH}}}{10^{9} \mathrm{GeV}}\right)+\frac{2}{3} \ln \left(\frac{\rho_{\mathrm{inf}}^{1 / 4}}{10^{16} \mathrm{GeV}}\right) .
$$

As we will see in Sec. III the Hubble parameter $H_{k}$ is related to the amplitude $\mathcal{P}_{T}$ of tensor perturbations as $\mathcal{P}_{T}=2 H_{k}^{2} /\left(\pi^{2} M_{\mathrm{pl}}^{2}\right)=r \mathcal{P}_{\mathcal{R}}$, where $\mathcal{P}_{\mathcal{R}} \simeq 2.198 \times 10^{-9}$ is the amplitude of curvature perturbations constrained by Planck [6]. Then, the inflationary energy scale can be generally estimated as

$$
\rho_{\text {inf }}^{1 / 4}=1.84 \times 10^{16}\left(\frac{r}{0.1}\right)^{1 / 4} \mathrm{GeV}
$$

For the monomial potential $V(\phi)=\lambda \phi^{n} / n$ the field value during inflation is analytically known as $\phi \simeq$ $\sqrt{2 n(N+n / 4)} M_{\mathrm{pl}}$, with $\phi_{\mathrm{end}}=n M_{\mathrm{pl}} / \sqrt{2}$. Hence the parameter $\alpha$ can be estimated as

$$
\alpha \simeq \frac{4}{3} \frac{\phi_{\mathrm{end}}^{n}}{\phi^{n}} \simeq \frac{4}{3}\left(\frac{n}{4 N}\right)^{n / 2}
$$

where the factor $4 / 3$ comes from the contribution of the inflaton kinetic energy to $\rho_{\text {end }}$. For the model (i), i.e., $n=2$, we have $\alpha \simeq 2 /(3 N)$. This leads to a non-negligible change to $N(k)$ of the order of 1 . Accordingly, Eq. (20) becomes

$$
N(k)=56.5+\frac{1}{3} \ln N(k)-\ln \left(\frac{k}{a_{0} H_{0}}\right)-\ln \left(\frac{h}{0.67}\right)+\frac{1}{3} \ln \left(\frac{T_{\mathrm{RH}}}{10^{9} \mathrm{GeV}}\right)+\frac{1}{6} \ln \left(\frac{r}{0.1}\right) .
$$

For given $k, h$, and $T_{\mathrm{RH}}, N(k)$ is known by solving Eq. (23). In Fig. 1, we plot $N(k)$ versus $T_{\mathrm{RH}}$ for several different values of $k$. This result is obtained by numerically solving the background equations of motion from inflation to the present epoch. We have confirmed that the numerical values of $N(k)$ show good agreement with those estimated by Eq. (23). On the largest scale observed in the CMB $\left(k=a_{0} H_{0}\right)$, the number of e-foldings is in the range $53<N(k)<61$ for $10^{3} \mathrm{GeV}<T_{\mathrm{RH}}<10^{13} \mathrm{GeV}$. For larger $k, N(k)$ becomes smaller.

In natural inflation (the model (ii)), the number of e-foldings can also be calculated as

$$
N \simeq-2\left(\frac{f}{M_{\mathrm{pl}}}\right)^{2} \ln \left[\sqrt{1+\frac{1}{2}\left(\frac{M_{\mathrm{pl}}}{f}\right)^{2}} \cos \left(\frac{\phi}{2 f}\right)\right],
$$

with $\cos ^{2}\left(\phi_{\mathrm{end}} / 2 f\right)=1 /\left[1+\left(M_{\mathrm{pl}} / f\right)^{2} / 2\right]$. Then, the parameter $\alpha$ reads

$$
\alpha \simeq \frac{4}{3} \frac{\sin ^{2}\left(\phi_{\mathrm{end}} / 2 f\right)}{\sin ^{2}(\phi / 2 f)} \simeq \frac{4 / 3}{1+2\left(f / M_{\mathrm{pl}}\right)^{2}\left(1-e^{-N M_{\mathrm{pl}}^{2} / f^{2}}\right)}
$$

Accordingly, Eq. (20) is replaced by

$$
\begin{aligned}
N(k)= & 56.5+\frac{1}{3} \ln \left[\frac{1}{2}+\left(\frac{f}{M_{\mathrm{pl}}}\right)^{2}\left(1-e^{-N(k) M_{\mathrm{pl}}^{2} / f^{2}}\right)\right]-\ln \left(\frac{k}{a_{0} H_{0}}\right)-\ln \left(\frac{h}{0.67}\right) \\
& +\frac{1}{3} \ln \left(\frac{T_{\mathrm{RH}}}{10^{9} \mathrm{GeV}}\right)+\frac{1}{6} \ln \left(\frac{r}{0.10}\right) .
\end{aligned}
$$

In the $f \rightarrow \infty$ limit, natural inflation becomes indistinguishable from quadratic chaotic inflation, so that Eq. (26) reduces to Eq. (23).

In Fig. 11, we show numerically derived values of $N(k)$ versus $T_{\mathrm{RH}}$ for the model (ii) with $f=7 M_{\mathrm{pl}}$. The number of e-foldings exhibits only a tiny difference from that in the model (i). 


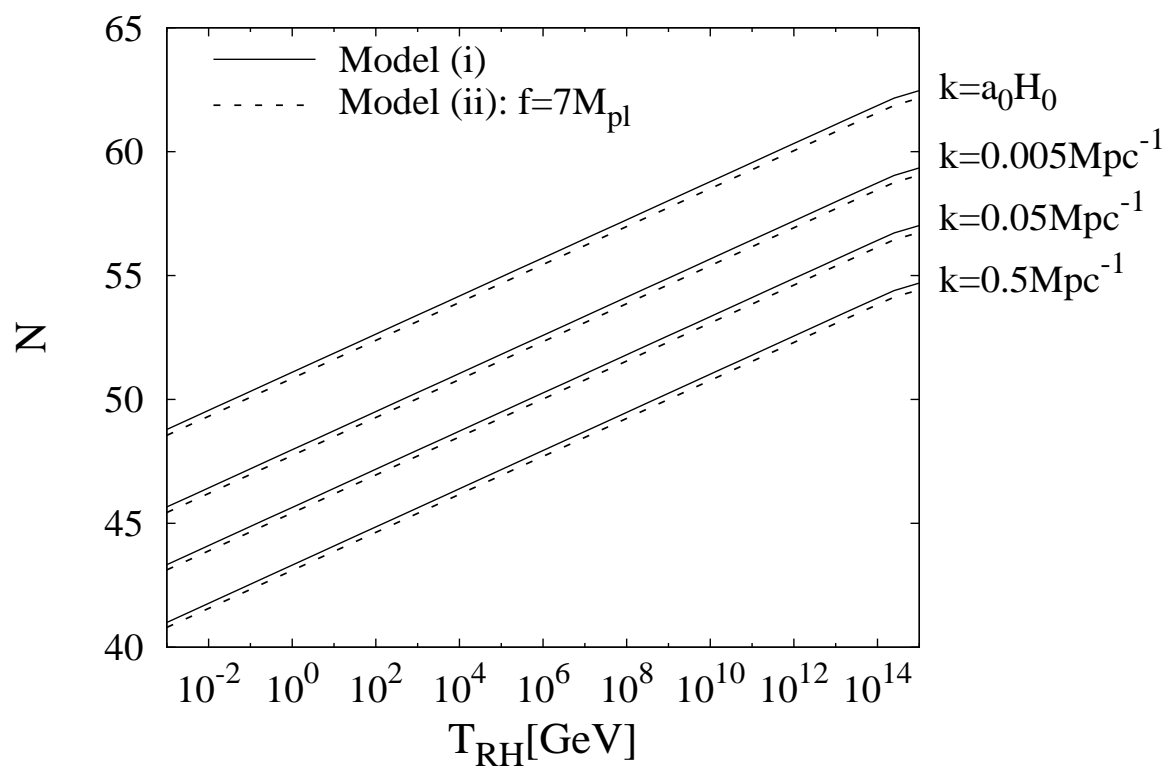

Figure 1: The number of e-foldings $N(k)$ versus the reheating temperature $T_{\mathrm{RH}}$ for the model (i) (solid line) and the model (ii) with $f=7 M_{\mathrm{pl}}$ (dashed line). We choose four different values of $k$ which are inside the observed CMB range. For increasing $T_{\mathrm{RH}}$ and decreasing $k, N(k)$ gets larger.

\section{Massless inflaton $(q=4)$}

For $|\xi| \ll 1$ the model (iii) belongs to the class of massless inflaton characterized by $q=4$. In this case, the number of e-foldings (18) does not depend on $T_{\mathrm{RH}}$. Following the same argument as before and using the relations (21) and (22) with $n=4$, we obtain

$$
N(k)=61.6+\frac{1}{2} \ln N(k)-\ln \left(\frac{k}{a_{0} H_{0}}\right)-\ln \left(\frac{h}{0.67}\right)-\frac{1}{12} \ln \left(\frac{g_{*, \mathrm{RH}}}{106.75}\right)+\frac{1}{4} \ln \left(\frac{r}{0.1}\right) .
$$

If $h=0.67, g_{*, \mathrm{RH}}=106.75$ and $r=0.1$, we have $N(k)=63.6$ for $k=a_{0} H_{0}$ and $N(k)=58.2$ for $k=0.05 \mathrm{Mpc}^{-1}$.

\section{PRIMORDIAL PERTURBATIONS GENERATED IN LARGE-FIELD INFLATIONARY MODELS AND CONSTRAINTS FROM CMB}

We consider the following perturbed metric on the flat FLRW background [47]:

$$
d s^{2}=-(1+2 A) d t^{2}+2 \partial_{i} B d t d x^{i}+a^{2}(t)\left[(1+2 \psi) \delta_{i j}+h_{i j}\right] d x^{i} d x^{j},
$$

where $A, B$, and $\psi$ are scalar perturbations, and $h_{i j}$ is the tensor perturbation. We choose the unitary gauge in which the field perturbation $\delta \phi$ vanishes, such that the gauge-invariant curvature perturbation $\mathcal{R} \equiv \psi-H \delta \phi / \dot{\phi}$ is identical to $\psi$. This fixes the time component of the gauge-transformation vector $\xi^{\mu}$. We have also fixed the spatial component of $\xi^{\mu}$ by gauging away the scalar perturbation $E$ appearing as the form $E_{, i j}$ in the metric (28).

\section{A. Tensor perturbations}

Expanding the action (11) for the transverse and traceless tensor perturbation, the second-order action reads

$$
S_{t}^{(2)}=\int d^{4} x a^{3} \frac{M_{\mathrm{pl}}^{2} F}{4}\left[\frac{1}{2} \dot{h}_{i j}^{2}-\frac{1}{2 a^{2}}\left(\partial h_{i j}\right)^{2}\right],
$$


where $F \equiv 1-\xi \phi^{2} / M_{\mathrm{pl}}^{2}$. We write $h_{i j}$ in terms of Fourier components, as

$$
h_{i j}(t, \boldsymbol{x})=\sum_{\mu=+, \times} \int \frac{d^{3} k}{(2 \pi)^{3 / 2}} \epsilon_{i j}^{\mu}(\boldsymbol{k}) h_{\boldsymbol{k}}^{\mu}(t) e^{i \boldsymbol{k} \cdot \boldsymbol{x}},
$$

where $\boldsymbol{k}$ is a comoving wave number. The polarization tensors $\epsilon_{i j}^{+, \times}$satisfy symmetric and transverse-traceless conditions and are normalized as $\sum_{i, j} \epsilon_{i j}^{\mu}\left(\epsilon_{i j}^{\mu^{\prime}}\right)^{*}=2 \delta^{\mu \mu^{\prime}}$. From the action (29), the Fourier mode $h_{\boldsymbol{k}}^{\mu}$ obeys the equation of motion

$$
\ddot{h}_{\boldsymbol{k}}^{\mu}+\left(3 H+\frac{\dot{F}}{F}\right) \dot{h}_{\boldsymbol{k}}^{\mu}+\frac{k^{2}}{a^{2}} h_{\boldsymbol{k}}^{\mu}=0 .
$$

For the quantization procedure, we express $h_{\boldsymbol{k}}^{\mu}$ in the form

$$
h_{\boldsymbol{k}}^{\mu}(t)=h_{k}(t) a_{\mu}(\boldsymbol{k})+h_{k}^{*}(t) a_{\mu}^{\dagger}(-\boldsymbol{k})
$$

where the annihilation and creation operators satisfy $\left[a_{\mu}(\boldsymbol{k}), a_{\mu^{\prime}}^{\dagger}\left(\boldsymbol{k}^{\prime}\right)\right]=\delta_{\mu \mu^{\prime}} \delta^{(3)}\left(\boldsymbol{k}-\boldsymbol{k}^{\prime}\right)$. A canonically normalized field, which is defined by

$$
v_{k} \equiv z h_{k}, \quad \text { where } \quad z \equiv a M_{\mathrm{pl}} \sqrt{F / 2}
$$

obeys the equation of motion

$$
v_{k}^{\prime \prime}+\left(k^{2}-\frac{z^{\prime \prime}}{z}\right) v_{k}=0
$$

where a prime represents a derivative with respect to the conformal time $\tau \equiv \int a^{-1} d t$. In the asymptotic past, the solution corresponding to the Bunch-Davies vacuum is given by $v_{k}=e^{-i k \tau} / \sqrt{2 k}$, i.e.,

$$
h_{k}=\frac{e^{-i k \tau}}{a M_{\mathrm{pl}} \sqrt{F k}}, \quad \text { for } \quad \tau \rightarrow-\infty .
$$

On the quasi de Sitter background with a nearly constant Hubble parameter, the variation of the quantity $F$ is negligibly small relative to that of $a$, such that $z^{\prime \prime} / z \simeq a^{\prime \prime} / a \simeq 2 / \tau^{2}$. Under this approximation, the solution to Eq. (34) during inflation reads

$$
h_{k}(\tau) \simeq \frac{i H e^{-i k \tau}}{k^{3 / 2} M_{\mathrm{pl}} \sqrt{F}}(1+i k \tau)
$$

We define the tensor power spectrum, as

$$
\mathcal{P}_{T} \equiv \frac{k^{3}}{\pi^{2}} \sum_{\mu=+, \times}\left|h_{\boldsymbol{k}}^{\mu}\right|^{2}
$$

After the Hubble radius crossing $(k=a H)$, the solution (36) approaches $h_{k} \rightarrow i H /\left(k^{3 / 2} M_{\mathrm{pl}} \sqrt{F}\right)$ so that we obtain the primordial power spectrum

$$
\left.\mathcal{P}_{T}^{\text {prim }} \simeq \frac{2 H^{2}}{\pi^{2} M_{\mathrm{pl}}^{2} F}\right|_{k=a H}
$$

The formula (38) has been derived under the condition that the variations of $H$ and $F$ are negligible during inflation. This is a good approximation for the perturbations relevant to CMB anisotropies $(50 \sim 60$ e-foldings before the end of inflation), but it is not so for the modes associated with the direct detection of gravitational waves (10 $\sim 20$ e-foldings before the end of inflation). For precise computations of the spectrum of the gravitational wave background, we shall numerically solve Eq. (31) under the initial condition (35) without using the formula (38). 


\section{B. Scalar perturbations and the tensor-to-scalar ratio}

For the study of scalar perturbations and the tensor-to-scalar ratio, it is convenient to perform the so-called conformal transformation $\hat{g}_{\mu \nu}=\Omega g_{\mu \nu}$, where a hat is used for quantities in the new frame and $\Omega$ is a conformal factor. For the choice $\Omega=F(\phi)$, the action (11) transforms to [48]

$$
\hat{S}=\int d^{4} x \sqrt{-\hat{g}}\left[\frac{1}{2} M_{\mathrm{pl}}^{2} \hat{R}-\frac{1}{2} \hat{g}^{\mu \nu} \partial_{\mu} \chi \partial_{\nu} \chi-U(\chi)\right],
$$

where

$$
U=\frac{V}{F^{2}}, \quad \chi \equiv \int \mathcal{B}(\phi) d \phi, \quad \mathcal{B}(\phi) \equiv \sqrt{\frac{3}{2}\left(\frac{M_{\mathrm{pl}} F_{, \phi}}{F}\right)^{2}+\frac{1}{F}}
$$

The perturbed metric in the Einstein frame (39) is given by $d \hat{s}^{2}=F d s^{2}$, where $d s^{2}$ corresponds to the metric (28). Decomposing the quantity $F$ into the background and perturbed components as $F(t)+\delta F(t, \boldsymbol{x})$, we obtain the following correspondence:

$$
\begin{aligned}
& \hat{a}=a \sqrt{F}, \quad \hat{t}=\int \sqrt{F} d t, \quad \hat{H}=\frac{1}{\sqrt{F}}\left(H+\frac{\dot{F}}{2 F}\right), \\
& \hat{A}=A+\frac{\delta F}{2 F}, \quad \hat{B}=B, \quad \hat{\psi}=\psi+\frac{\delta F}{2 F}, \quad \hat{h}_{i j}=h_{i j},
\end{aligned}
$$

so that the tensor perturbation is invariant under the conformal transformation. On using the relations (41), the power spectrum (38) reads

$$
\left.\mathcal{P}_{T}^{\text {prim }} \simeq \frac{2 \hat{H}^{2}}{\pi^{2} M_{\mathrm{pl}}^{2}}\right|_{k=\hat{a} \hat{H}}
$$

at leading order in slow-roll (under which the term $\dot{F} /(2 F)$ is negligible relative to $H$ ).

Using the correspondence (42), one can show that the curvature perturbation $\mathcal{R}=\psi-H \delta \phi / \dot{\phi}$ is invariant under the conformal transformation [24, 25]. In the Einstein frame the power spectrum of $\mathcal{R}$ is equivalent to the one in standard slow-roll inflation, so it is given by [7]

$$
\left.\mathcal{P}_{\mathcal{R}}^{\text {prim }} \simeq \frac{\hat{H}^{2}}{8 \pi^{2} \epsilon_{U} M_{\mathrm{pl}}^{2}}\right|_{k=\hat{a} \hat{H}}
$$

at leading order in slow-roll, where $\epsilon_{U} \equiv\left(M_{\mathrm{pl}}^{2} / 2\right)\left(U_{, \chi} / U\right)^{2}$. The scalar spectral index $n_{s} \equiv 1+d \ln \mathcal{P}_{\mathcal{R}}^{\text {prim }} /\left.d \ln k\right|_{k=\hat{a} \hat{H}}$ and the tensor-to-scalar ratio $r=\mathcal{P}_{T}^{\text {prim }} /\left.\mathcal{P}_{\mathcal{R}}^{\text {prim }}\right|_{k=\hat{a} \hat{H}}$ are

$$
n_{s}=1-6 \epsilon_{U}+2 \eta_{U}, \quad r=-8 n_{t}=16 \epsilon_{U},
$$

where $\eta_{U} \equiv M_{\mathrm{pl}}^{2} U, \chi \chi / U$ and $n_{t} \equiv d \ln \mathcal{P}_{T}^{\text {prim }} /\left.d \ln k\right|_{k=\hat{a} \hat{H}}$ is the tensor spectral index. For a given inflaton potential, these observables can be explicitly expressed as a function of $\phi$ by using the relations (40).

\section{Observational implications from the recent CMB data}

We compute the CMB observables (45) for the models (i)-(iii) to confront them with observations. We refer the readers to Refs. [10] for detailed calculations of $n_{s}$ and $r$. The number of e-foldings during inflation can be expressed as the integrated form $N(t)=-\int_{t_{f}}^{t} H(\tilde{t}) d \tilde{t}$ in the Jordan frame. With a proper choice of a reference length scale, the number of e-foldings is a frame-independent quantity [49]. From the third relation of Eq. (41), it follows that

$$
N=\int_{\chi_{f}}^{\chi} \frac{U}{M_{\mathrm{pl}}^{2} U_{, \chi}} d \chi+\frac{1}{2} \ln \frac{F(t)}{F\left(t_{f}\right)}
$$

where we have employed the slow-roll approximation in the Einstein frame. 

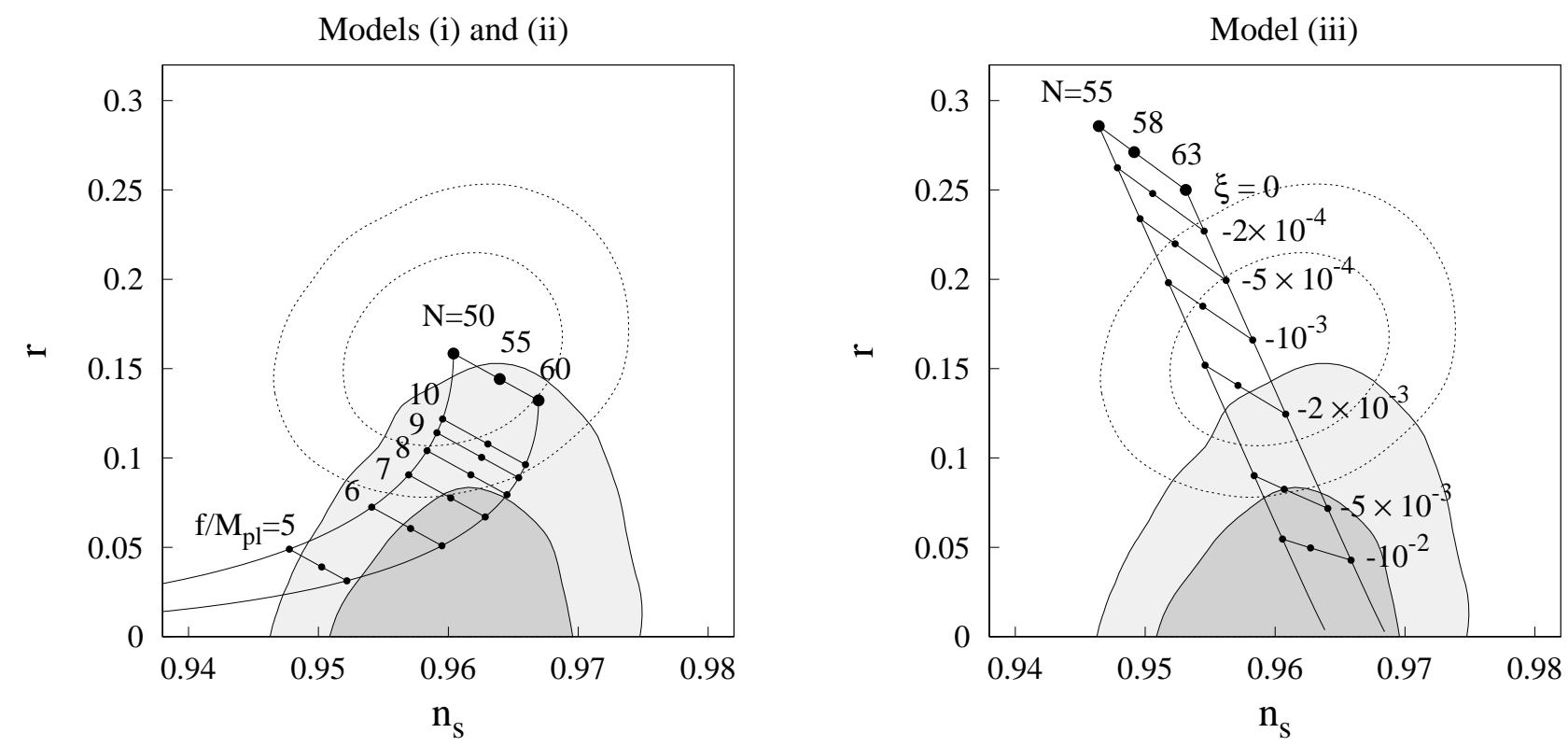

Figure 2: Two-dimensional observational constraints on the models (i) and (ii) (left), and the model (iii) (right) in the $\left(n_{s}, r\right)$ plane with the pivot wave number $k_{*}=0.05 \mathrm{Mpc}^{-1}$. The gray shaded regions represent the $68 \%$ C.L. (inside) and $95 \%$ C.L. (outside) parameter spaces constrained by the joint data analysis of Planck+WP+BAO+high- $\ell$. We also show the $68 \%$ C.L. (inside) and $95 \%$ C.L. (outside) boundaries constrained by Planck+WP+BAO+high- $\ell+$ BICEP2 with dotted ellipses. In the left panel, the three large black points correspond to the theoretical prediction of the model (i) for $N=50,55,60$, whereas the small black points represent the prediction of the model (ii) for $f / M_{\mathrm{pl}}=5,6,7,8,9,10$ with $N=50,55,60$. In the right panel, the theoretical curves are plotted for $N=55,58,63$ with several different values of $\xi$ between 0 and $-10^{-2}$.

For the CMB likelihood analysis, we expand the primordial power spectra $\mathcal{P}_{\mathcal{R}}^{\text {prim }}$ and $\mathcal{P}_{T}^{\text {prim }}$ as a Taylor series about a pivot wave number $k_{*}$. We employ the consistency relation $r=-8 n_{t}$ and set the scalar and tensor runnings to be 0 in the likelihood analysis. The likelihood results are derived with the CosmoMC code [50, 51] by assuming the flat $\Lambda$ CDM model and $N_{\text {eff }}=3.046$ relativistic degrees of freedom with an instant reionization.

In Fig. 2 we plot the $68 \%$ C.L. and $95 \%$ C.L. regions (plotted as the gray shaded color) in the $\left(n_{s}, r\right)$ plane constrained by the joint data analysis of Planck [6], WP [13], baryon acoustic oscillations (BAO) [52], and Atacama Cosmology Telescope/South Pole Telescope temperature data of high multipoles $\ell$ (high- $\ell$ ) [53]. The tensor-to-scalar ratio is constrained to be $r<0.15$ at $95 \%$ C.L. We have chosen the value $k_{*}=0.05 \mathrm{Mpc}^{-1}$ in Fig. 2, but the results are insensitive to the choice of $k_{*}$ as long as the pivot scale is in the observed range of CMB. Hence, the likelihood contours for different choices of $k_{*}$ are similar to those given in Fig. 2, We also show the 68 \% C.L. and $95 \%$ C.L. boundaries (dotted curves) constrained by adding the BICEP2 data [12] to the Planck+WP+BAO+high- $\ell$ data. In this case, $r$ is bounded from below.

For the monomial potential $V(\phi)=\lambda \phi^{n} / n$ with $\xi=0$, the observables (46) reduce to [7]

$$
n_{s}=1-\frac{2(n+2)}{4 N+n}, \quad r=\frac{16 n}{4 N+n} .
$$

From Eq. (23), the number of e-foldings for the quadratic potential $(n=2)$ depends on the reheating temperature $T_{\mathrm{RH}}$ and the wave number $k$. For the pivot scale $k_{*}=0.05 \mathrm{Mpc}^{-1}$ the number of e-foldings is in the range $48.7<N\left(k_{*}\right)<$ 53.3 for $10^{4} \mathrm{GeV}<T_{\mathrm{RH}}<10^{10} \mathrm{GeV}$. If $k_{*}=a_{0} H_{0}$, then $54.1<N\left(k_{*}\right)<58.8$ for $10^{4} \mathrm{GeV}<T_{\mathrm{RH}}<10^{10} \mathrm{GeV}$. In Fig. 2. we plot the theoretical curves of the model (i) in the $\left(n_{s}, r\right)$ plane for $N$ between 50 and 60 . For $N>52$ the quadratic potential is inside the $95 \%$ C.L. boundary constrained by the Planck+WP+BAO+high- $\ell$ data.

In Fig. 2, we also show the theoretical prediction of the model (ii) for $N=50,55,60$ with $f$ between $5 M_{\mathrm{pl}}$ and $10 M_{\mathrm{pl}}$. When $N>50$, natural inflation is inside the $95 \%$ C.L. boundary constrained the Planck $+\mathrm{WP}+\mathrm{BAO}+$ high- $\ell$ data for $f>5 M_{\mathrm{pl}}$. In the $f \rightarrow \infty$ limit, inflation occurs in the region around the potential minimum $(\phi=\pi f)$, in which case $n_{s}$ and $r$ approach the values (47) with $n=2$.

In the model (iii) with $|\xi| \ll 1$, the number of e-foldings (27) does not depend on $T_{\mathrm{RH}}$. For given $k_{*}$, the value of $N$ is fixed, e.g., $N=58$ for $k_{*}=0.05 \mathrm{Mpc}^{-1}$. In the right panel of Fig. 2, we plot the theoretical values of $n_{s}$ 
and $r$ for $N=55,58,60$ with several different values of $\xi$. For $|\xi|$ of the order of $10^{-3}$, the presence of non-minimal couplings allows a possibility of reducing the values of $r$ to be compatible with the upper bound derived from the Planck+WP+BAO+high- $\ell$ data. In the $|\xi| \rightarrow \infty$ limit, we obtain the same values of $n_{s}$ and $r$ as those in the Starobinsky $f(R)$ model [54], i.e., $n_{s}=1-2 / N$ and $r=12 / N^{2}$ [25].

\section{THE INTENSITY OF THE GRAVITATIONAL WAVE BACKGROUND}

In this section, we calculate the spectrum of the gravitational wave background generated in large-field inflationary models discussed in Sec. III. From the second-order action (29), the energy density $\rho_{\mathrm{GW}}$ of gravitational waves corresponds to the (00) component of its energy-momentum tensor $T_{\mu \nu}$, i.e.,

$$
\rho_{\mathrm{GW}}=\frac{M_{\mathrm{pl}}^{2} F}{8 a^{2}}\left[\left(h_{i j}^{\prime}\right)^{2}+\left(\partial h_{i j}\right)^{2}\right] .
$$

After the perturbations enter the Hubble radius during the radiation or matter era, the WKB solution to Eq. (31) is given by

$$
h_{\boldsymbol{k}}^{\mu} \propto a^{-1} e^{ \pm i k \tau},
$$

where we have neglected the variation of $F$ relative to that of $a$. Substituting Eq. (30) with the solution (49) into Eq. (48) and taking the spatial average, the energy density reads

$$
\rho_{\mathrm{GW}}=\frac{M_{\mathrm{pl}}^{2} F}{2} \int \frac{d^{3} k}{(2 \pi)^{3}} \frac{k^{2}}{a^{2}} \sum_{\mu=+, \times}\left|h_{\boldsymbol{k}}^{\mu}\right|^{2} .
$$

We define the intensity of the gravitational wave background as

$$
\Omega_{\mathrm{GW}} \equiv \frac{1}{\rho_{c}} \frac{d \rho_{\mathrm{GW}}}{d \ln k}=\frac{1}{12}\left(\frac{k}{a H}\right)^{2} \mathcal{P}_{T},
$$

where $\rho_{c} \equiv 3 F H^{2} M_{\mathrm{pl}}^{2}$ is the critical density of the Universe, and $\mathcal{P}_{T}$ is the tensor spectrum defined in Eq. (37). In the second equality of Eq. (51), we have substituted the energy density (50).

In order to compute the spectrum (51) today, we need to solve Eq. (31) from the onset of inflation to the present epoch. As we have already studied in Sec. IIII, the background dynamics during inflation and reheating are known by solving Eqs. (7)-(9) numerically. To discuss the cosmological dynamics after the radiation era, we need to take into account the contribution of relativistic particles, non-relativistic particles (dark matter and baryons), and dark energy to the Friedmann equation.

The entropy density of relativistic particles at temperature $T$ is given by $s(T)=\left(2 \pi^{2} / 45\right) g_{s}(T) T^{3}$. Due to the entropy conservation $s a^{3}=$ constant, the radiation energy density $\rho_{r}(T)=\left(\pi^{2} / 30\right) g_{*}(T) T^{4}$ evolves as $\rho_{r} \propto g_{*} g_{s}^{-4 / 3} a^{-4}$. The explicit forms of $g_{*}(T)$ and $g_{s}(T)$ are given in Ref. [55]. The energy density of non-relativistic particles decreases as $\rho_{m} \propto a^{-3}$. For dark energy, we assume that its energy density $\rho_{\mathrm{DE}}$ is given by the cosmological constant $\Lambda$. We can consider some other sources for dark energy, but it hardly affects the resulting gravitational wave spectrum unless Eq. (31) is subject to change under some modification of gravity. The term $F$ is very close to 1 around today, so the effect of non-minimal couplings is negligibly small at the late cosmological epoch.

Defining today's density parameters as $\Omega_{j 0}=\rho_{j 0} /\left(3 F_{0} H_{0}^{2} M_{\mathrm{pl}}^{2}\right)$, where $j=r, m, \Lambda$ and the subscript "0" represents the present values, the Friedmann equation after the end of reheating can be expressed as

$$
\frac{H^{2}}{H_{0}^{2}}=\frac{F_{0}}{F}\left[\left(\frac{g_{*}}{g_{* 0}}\right)\left(\frac{g_{s}}{g_{s 0}}\right)^{-4 / 3} \Omega_{r 0}\left(\frac{a}{a_{0}}\right)^{-4}+\Omega_{m 0}\left(\frac{a}{a_{0}}\right)^{-3}+\Omega_{\Lambda 0}\right] \text {. }
$$

For numerical simulations, we take the present radiation density to be $\Omega_{r 0} h^{2}=4.15 \times 10^{-5}$ and use the mean likelihood values derived from the Planck $+\mathrm{WP}+\mathrm{BAO}+$ high- $\ell$ data [6]: the non-relativistic matter density $\Omega_{m 0} h^{2}=0.141$, the dark energy density $\Omega_{\Lambda 0}=0.692$, the amplitude of primordial curvature perturbations $\mathcal{P}_{\mathcal{R}}^{\text {prim }}=2.2 \times 10^{-9}$, and the normalized Hubble constant $h=0.678$. For the calculation of relativistic degrees of freedom, we only include particles in the standard model of particle physics, where the maximum values of $g_{*}$ and $g_{s}$ are 106.75.

Since the primordial tensor perturbation is frozen by the second horizon crossing characterized by $k=a_{\mathrm{hc}} H_{\mathrm{hc}}$, today's power spectrum $\mathcal{P}_{T 0}$ is related to the primordial one $\mathcal{P}_{T}^{\text {prim }}$ via $\mathcal{P}_{T 0}=\mathcal{P}_{T}^{\text {prim }}\left(a_{\mathrm{hc}} / a_{0}\right)^{2}$. If the scale factor 

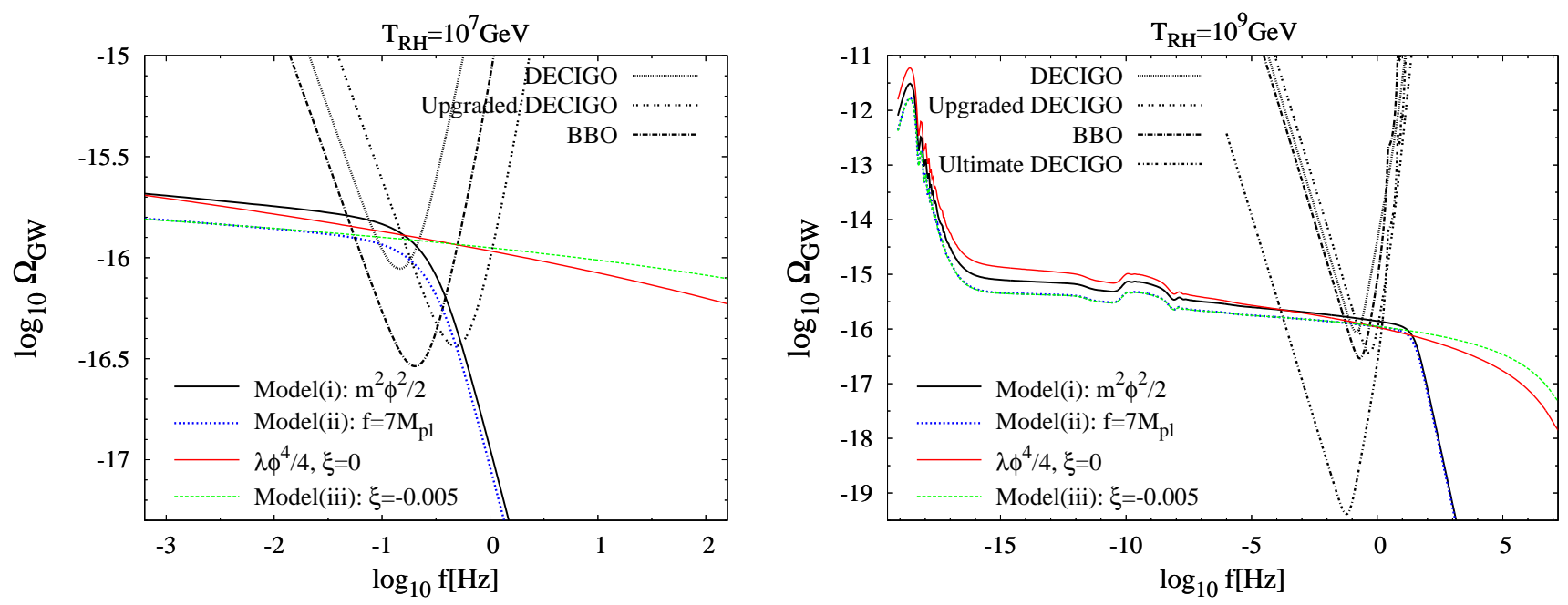

Figure 3: Today's intensity $\Omega_{\mathrm{GW}}$ of the gravitational wave background versus the frequency $f=k /(2 \pi)$ for the four models: (i) $V(\phi)=m^{2} \phi^{2} / 2$ with $\xi=0$, (ii) $V(\phi)=\Lambda^{4}[1+\cos (\phi / f)]$ with $\xi=0$ and $f=7 M_{\mathrm{pl}}$, the potential $V(\phi)=\lambda \phi^{4} / 4$ with $\xi=0$, and (iii) $V(\phi)=\lambda \phi^{4} / 4$ and $\xi=-5.0 \times 10^{-3}$. We also show the sensitivity curves for DECIGO, upgraded DECIGO, BBO, and Ultimate DECIGO. Each panel corresponds to the spectra around the detection sensitivity with $T_{\mathrm{RH}}=10^{7} \mathrm{GeV}$ (left) and the spectra in the wide range of frequencies with $T_{\mathrm{RH}}=10^{9} \mathrm{GeV}$ (right).

evolves as $a \propto t^{p}$, where $p$ is a constant, the Hubble parameter $H$ is proportional to $a^{-1 / p}$, so that $a_{\mathrm{hc}} \propto k^{p /(p-1)}$. Then, today's gravitational wave intensity (51) has the momentum dependence

$$
\Omega_{\mathrm{GW}} \propto k^{n_{t}+2(2 p-1) /(p-1)},
$$

where $n_{t}$ is the primordial tensor spectral index.

During the radiation era $(p=1 / 2)$ and the matter era $(p=2 / 3)$, we have $\Omega_{\mathrm{GW}} \propto k^{n_{t}}$ and $\Omega_{\mathrm{GW}} \propto k^{n_{t}-2}$, respectively. The nearly scale-invariant property of the primordial tensor perturbation is imprinted for the modes that re-entered the Hubble radius during the radiation-dominated epoch. This is the case for the frequencies $f=k /(2 \pi)$ associated with the direct detection of gravitational waves by DECIGO or BBO $(\sim 1 \mathrm{~Hz})$. For the perturbations that re-entered the Hubble radius during the matter era, the intensity $\Omega_{\mathrm{GW}}$ has a highly red-tilted spectrum.

For the inflationary models like (i) and (ii), the reheating stage corresponds to a temporal matter-dominated epoch driven by a massive inflation field. Then, there is a suppression of $\Omega_{\mathrm{GW}}$ at high frequencies. This suppression occurs for $[56]$

$$
f>f_{\mathrm{RH}} \equiv 0.26\left(\frac{T_{\mathrm{RH}}}{10^{7} \mathrm{GeV}}\right)\left(\frac{g_{*, \mathrm{RH}}}{100}\right)^{1 / 2}\left(\frac{g_{s, \mathrm{RH}}}{100}\right)^{-1 / 3} \mathrm{~Hz} .
$$

For increasing $\Gamma$, the critical frequency $f_{\mathrm{RH}}$ becomes larger. In the model (iii) with $|\xi| \ll 1$, the evolution of the scale factor during reheating is given by $a \propto t^{1 / 2}$, so the suppression of $\Omega_{\mathrm{GW}}$ mentioned above is absent. As we explained in Sec. II] we compute the reheating temperature numerically at the time when the field energy density $\rho_{\phi}$ drops below the radiation energy density $\rho_{r}$.

Under the slow-roll approximation the primordial spectral index is given by $n_{t}=-2 \epsilon_{U}$. Note, however, that this formula overestimates the amplitude of the gravitational wave spectrum at $f \sim 1 \mathrm{~Hz}$ by $20 \%$ for chaotic inflation models [55]. Therefore, we compute the intensity $\Omega_{\mathrm{GW}}$ for three inflationary models (i)-(iii) without employing the slow-roll approximation. We numerically solve Eq. (31) for each wave number $k$ together with the background equations of motion (77)-(9) during inflation/reheating and Eq. (52) after the end of reheating.

In Fig. 3. we show today's gravitational wave intensity versus the frequency $f$ for the three large-field inflationary models (i)-(iii) with $T_{\mathrm{RH}}=10^{7} \mathrm{GeV}$ (left) and $T_{\mathrm{RH}}=10^{9} \mathrm{GeV}$ (right). Recall that the reheating temperatures for the models (i) and (ii) are related to the number of e-foldings $N$, as shown in Fig. 1. In the left panel, we see that the anisotropic stress due to neutrino free streaming [37, 55, 57] at $f<10^{-9} \mathrm{~Hz}$ does not affect the amplitude of the gravitational waves at the direct detection scales $\left(\sim 10^{-1} \mathrm{~Hz}\right)$. Since the primordial tensor-to-scalar ratio in the model (i) is greater than that in the model (ii) (see Fig. (2), the amplitude of $\Omega_{\mathrm{GW}}$ in the former is larger than that in the latter at the CMB scale $\left(\sim 10^{-18} \mathrm{~Hz}\right)$. In Fig. 3 the suppression of $\Omega_{\mathrm{GW}}$ due to the presence of the temporal matter 
era after inflation is clearly seen for large frequencies. As estimated by Eq. (54), the critical frequency $f_{\mathrm{RH}}$ becomes larger for increasing $T_{\mathrm{RH}}$.

In the model (iii) with $|\xi| \ll 1$, the reheating stage is characterized by the oscillation of a massless inflaton, so the suppression of $\Omega_{\mathrm{GW}}$ mentioned above is not present. However, due to the steepness of the potential, the evolution of the inflaton is faster than that in the models (i) and (ii) around the end of inflation. As we see in the right panel of Fig. 3. this leads to the mild decrease of the power spectrum around the frequencies $f \gtrsim 10^{4} \mathrm{~Hz}$.

In Fig. 3, we also plot the sensitivity curves for DECIGO, upgraded DECIGO, BBO, and Ultimate DECIGO. In particular, the sensitivity of upgraded DECIGO is improved over DECIGO to cover a wider range of frequencies. In the model (i), BBO, and upgraded DECIGO can potentially measure inflationary gravitational waves for $T_{\mathrm{RH}} \gtrsim 10^{6}$ $\mathrm{GeV}$ and $T_{\mathrm{RH}} \gtrsim 10^{7} \mathrm{GeV}$, respectively. In the model (ii), the intensity $\Omega_{\mathrm{GW}}$ becomes smaller for decreasing $f$, but the detection is still possible for $f \gtrsim 4 M_{\mathrm{pl}}$. The model (iii) allows the possible detection in the frequency range $0.1 \mathrm{~Hz} \lesssim f \lesssim 1 \mathrm{~Hz}$, irrespective of the reheating temperature. In the next section, we shall discuss this issue in more detail by taking into account the noise associated with the interferometric detectors.

\section{DETECTABILITY OF INFLATIONARY GRAVITATIONAL WAVES}

In order to discuss the detectability of inflationary gravitational waves, we compute the signal-to-noise ratio (SNR) by two interferometric detectors. Here the SNR is expressed in terms of $\Omega_{\mathrm{GW}}(f)$ given by Eq. (51) and the functions related to the experimental design, such as the noise spectrum $N_{I, J}(f)$ and the overlap reduction function $\gamma_{I J}(f)$, as [58]

$$
[\mathrm{SNR}]^{2}=2\left(\frac{3 H_{0}^{2}}{10 \pi^{2}}\right)^{2} T_{\mathrm{obs}} \sum_{(I, J)} \int_{0}^{\infty} d f \frac{\left|\gamma_{I J}(f)\right|^{2} \Omega_{\mathrm{GW}}^{2}(f)}{f^{6} N_{I}(f) N_{J}(f)},
$$

where $T_{\text {obs }}$ is the duration of observations, and the subscripts $I$ and $J$ refer to independent signals obtained at each detector, or observables generated by combining the detector signals. The overlap reduction function $\gamma_{I J}(f)$ can be calculated with information about relative locations and orientations of detectors [59, 60]. In the following, we present explicit forms of the noise spectra for DECIGO, upgraded DECIGO, BBO, and Ultimate DECIGO.

\section{- DECIGO}

DECIGO is planned to be a Fabry-Perot Michelson interferometer with an arm length of $L=1.0 \times 10^{6} \mathrm{~m}[28]$. The noise spectral density is given by [59]

$$
N_{1}(f)=N_{2}(f)=S_{\text {shot }}^{2}+S_{\text {accel }}^{2}+S_{\text {rad }}^{2},
$$

where $S_{\text {shot }}=2.3 \times 10^{-24} \sqrt{\left(1+f^{2} / f_{c}^{2}\right)} \mathrm{Hz}^{-1 / 2}$ is the shot noise ${ }^{1}, S_{\text {accel }}=2.0 \times 10^{-26} /\left(f / \mathrm{Hz}^{2} \mathrm{~Hz}^{-1 / 2}\right.$ is the acceleration noise, and $S_{\mathrm{rad}}=6.0 \times 10^{-26} /\left[(f / \mathrm{Hz})^{2} \sqrt{1+f^{2} / f_{c}^{2}}\right] \mathrm{Hz}^{-1 / 2}$ is the radiation pressure noise, with $f_{c}=7.36 \mathrm{~Hz}$ being the cutoff frequency.

- Upgraded DECIGO

In order to resolve all foreground gravitational waves coming from neutron star binaries, it is necessary to improve the sensitivity of DECIGO by a factor of 3 [61]. We consider the following noise spectrum as an example of an upgraded version of DECIGO: $S_{\text {shot }}=3.3 \times 10^{-25} \sqrt{\left(1+f^{2} / f_{c}^{2}\right)} \mathrm{Hz}^{-1 / 2}$ and $S_{\text {accel }}=8.0 \times 10^{-26} /(f / \mathrm{Hz})^{2} \mathrm{~Hz}^{-1 / 2}$ with the cutoff frequency $f_{c}=3.57 \mathrm{~Hz}$, which would be possible by upgrading the arm length from $10^{6} \mathrm{~m}$ to $1.5 \times 10^{6} \mathrm{~m}$, the laser power from $10 \mathrm{~W}$ to $30 \mathrm{~W}$ and mirror radius from $0.5 \mathrm{~m}$ to $0.75 \mathrm{~m}$. For radiation pressure, we adopt the same noise spectrum as that of DECIGO, which is negligible compared to the acceleration noise.

- $\mathrm{BBO}$

BBO adopts a technique called Time-Delay Interferometry (TDI), in which new variables $(I=A, E, T)$ are constructed to cancel the laser frequency noise. The noise transfer functions for the TDI variables are given by [62]

$$
\begin{aligned}
& N_{A}(f)=N_{E}(f)=8 \sin ^{2}(\hat{f} / 2)\left\{(2+\cos \hat{f}) N_{\text {shot }}+2[3+2 \cos \hat{f}+\cos (2 \hat{f})] N_{\text {accel }}\right\}, \\
& N_{T}(f)=2(1+2 \cos \hat{f})^{2}\left[N_{\text {shot }}+4 \sin ^{2}(\hat{f} / 2) N_{\text {accel }}\right],
\end{aligned}
$$

\footnotetext{
${ }^{1}$ Here we have fixed a typo in Ref. [59] as already done in [38, 41].
} 


\begin{tabular}{lccccc}
\hline \hline Model & $r$ & $\Omega_{\mathrm{GW}}$ & SNR (DECIGO) & SNR (Upgraded DECIGO) & SNR (BBO) \\
\hline Model (i) & 0.153 & $1.55 \times 10^{-16}$ & 3.90 & 8.67 & 7.87 \\
Model (ii) $\left(f=10 M_{\mathrm{Pl}}\right)$ & 0.117 & $1.40 \times 10^{-16}$ & 3.52 & 6.81 & 17.2 \\
Model (ii) $\left(f=7 M_{\mathrm{Pl}}\right)$ & 0.086 & $1.21 \times 10^{-16}$ & 3.03 & 6.94 & 7.56 \\
$V(\phi)=\lambda \phi^{4} / 4(\xi=0)$ & 0.275 & $1.27 \times 10^{-16}$ & 3.24 & 6.90 \\
Model (iii) $(\xi=-0.002)$ & 0.144 & $1.36 \times 10^{-16}$ & 3.43 & 13.4 \\
Model (iii) $(\xi=-0.005)$ & 0.085 & $1.22 \times 10^{-16}$ & 3.06 & 15.1 \\
\hline \hline
\end{tabular}

Table I: The tensor-to-scalar ratio $r$ for the pivot wave number $k_{*}=0.05 \mathrm{Mpc}^{-1}$, the intensity of the gravitational wave $\Omega$ GW at the frequency $f=0.2 \mathrm{~Hz}$, and the SNR in DECIGO, upgraded DECIGO, and BBO for the three inflationary models listed in (2)-(4). The reheating temperature is set as $T_{\mathrm{RH}}=10^{9} \mathrm{GeV}$.

where $\hat{f}=2 \pi L f$. In the case of BBO, the arm length is $L=5.0 \times 10^{7} \mathrm{~m}$ and the noise functions are $N_{\text {shot }}=$ $2.0 \times 10^{-34} /(L / \mathrm{m})^{2} \mathrm{~Hz}^{-1}$ and $N_{\text {accel }}=9.0 \times 10^{-34} /\left[(2 \pi f / \mathrm{Hz})^{4} \cdot(2 L / \mathrm{m})^{2}\right] \mathrm{Hz}^{-1}$.

- Ultimate DECIGO

Ultimate DECIGO is an ideal experiment whose noise is limited only by quantum noise [28]. We consider a TDI type experiment whose arm length is $L=5.0 \times 10^{7} \mathrm{~m}$ and the noise functions are $N_{\text {shot }}=2.72 \times$ $10^{-36} /(L / \mathrm{m})^{2} \mathrm{~Hz}^{-1}$ and $N_{\text {accel }}=9.0 \times 10^{-38} /\left[(2 \pi f / \mathrm{Hz})^{4} \cdot(2 L / \mathrm{m})^{2}\right] \mathrm{Hz}^{-1}[59] .^{2}$

In Fig. 4 we show the SNR versus the reheating temperature $T_{\mathrm{RH}}$ for the cross-correlation analysis expected with 10-year observations by DECIGO, upgraded DECIGO, BBO, and Ultimate DECIGO. ${ }^{3}$ For the models (i) and (ii), the SNR decreases significantly for $T_{\mathrm{RH}}$ lower than $10^{7} \mathrm{GeV}$ because of the suppression due to the presence of the temporal matter era after inflation. The SNR of the model (ii) is smaller than that of the model (i) for the same reheating temperature. As we see in the left panel of Fig. [5 the SNR of natural inflation increases for larger $f$ to approach that of the model (i) in the $f \rightarrow \infty$ limit. This tendency is also seen in Table I in which the SNR as well as $r$ and $\Omega_{\mathrm{GW}}$ are given for $T_{\mathrm{RH}}=10^{9} \mathrm{GeV}$.

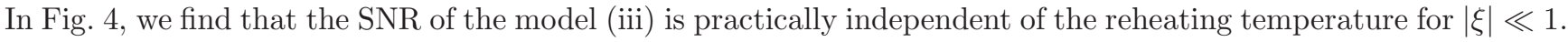
The primordial tensor-to-scalar ratio $r$ gets smaller for increasing $|\xi|$. As we see in the right panel of Fig. [5, the SNR starts to decrease for $\xi \lesssim-2 \times 10^{-3}$. This is inside the $95 \%$ C.L. region constrained by the Planck $+\mathrm{WP}+\mathrm{BAO}+$ high- $\ell$ data (see Fig. 2).

If the SNR is larger than 3, the primordial gravitational waves could be directly measured at $3 \sigma$ (under the assumption of Gaussian noise). For the model (i), the detections by DECIGO, upgraded DECIGO, BBO, and Ultimate DECIGO at $3 \sigma$ would be possible if the reheating temperature is larger than $7.8 \times 10^{6} \mathrm{GeV}, 7.9 \times 10^{6} \mathrm{GeV}$, $1.8 \times 10^{6} \mathrm{GeV}$, and $2.8 \times 10^{3} \mathrm{GeV}$, respectively. The detections by upgraded DECIGO (BBO) are also feasible at $5 \sigma$ if $T_{\mathrm{RH}}>1.5 \times 10^{7} \mathrm{GeV}\left(2.8 \times 10^{6} \mathrm{GeV}\right)$.

The situation in the model (ii) is similar to that in the model (i). For $f=7 M_{\mathrm{pl}}$, DECIGO, upgraded DECIGO and $\mathrm{BBO}$ could measure the primordial gravitational waves at $3 \sigma$ if $T_{\mathrm{RH}}>6.8 \times 10^{7} \mathrm{GeV}, T_{\mathrm{RH}}>9.9 \times 10^{6} \mathrm{GeV}$, $T_{\mathrm{RH}}>2.2 \times 10^{6} \mathrm{GeV}$, and $T_{\mathrm{RH}}>3.3 \times 10^{3} \mathrm{GeV}$, respectively. The detections would be possible at $5 \sigma$ for $T_{\mathrm{RH}}>$ $2.2 \times 10^{7} \mathrm{GeV}$ (upgraded DECIGO) and $T_{\mathrm{RH}}>2.2 \times 10^{7} \mathrm{GeV}$ (BBO). The SNR increases for larger $f$ and converges to that of the model (i). For $T_{\mathrm{RH}}>10^{8} \mathrm{GeV}$ the SNR is insensitive to the reheating temperature. The detections by upgraded DECIGO (BBO) would be possible for $f>4.2 M_{\mathrm{pl}}\left(f>3.6 M_{\mathrm{pl}}\right)$ at $3 \sigma$ and for $f>5.3 M_{\mathrm{pl}}\left(f>4.0 M_{\mathrm{pl}}\right)$ at $5 \sigma$. The $f$ dependence of the SNR for Ultimate DECIGO is the same as that of DECIGO/BBO seen in the left panel of Fig. 5 , but the scale of the vertical axis is much larger because of the extremely high sensitivity. Thus, Ultimate DECIGO can detect the signal with a very good accuracy as long as we consider the parameter region indicated by Planck, $f \gtrsim 5 M_{\mathrm{pl}}$.

The SNR in the model (iii) is independent of $T_{\mathrm{RH}}$, but it depends on non-minimal couplings $\xi$. From the right

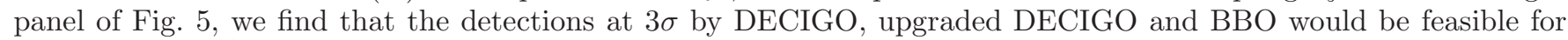
$\xi>-5.4 \times 10^{-3}, \xi>-0.035$ and $\xi>-0.1$, respectively. The $5 \sigma$ detections by upgraded DECIGO (BBO) would require $\xi>-0.013(-0.043)$. This corresponds to the regime in which the tensor-to-scalar ratio is larger than 0.05 .

\footnotetext{
2 Note that the shot noise is corrected from that of Ref. [59].

3 These SNRs are slightly larger than those computed in Ref. [4] by a factor $(220 / 106.75)^{1 / 3} \simeq 1.3$ because we consider the standard model particles alone and take $g_{*, \mathrm{RH}}=106.75$ rather than $g_{*, \mathrm{RH}}=220$ used in Ref. [41].
} 

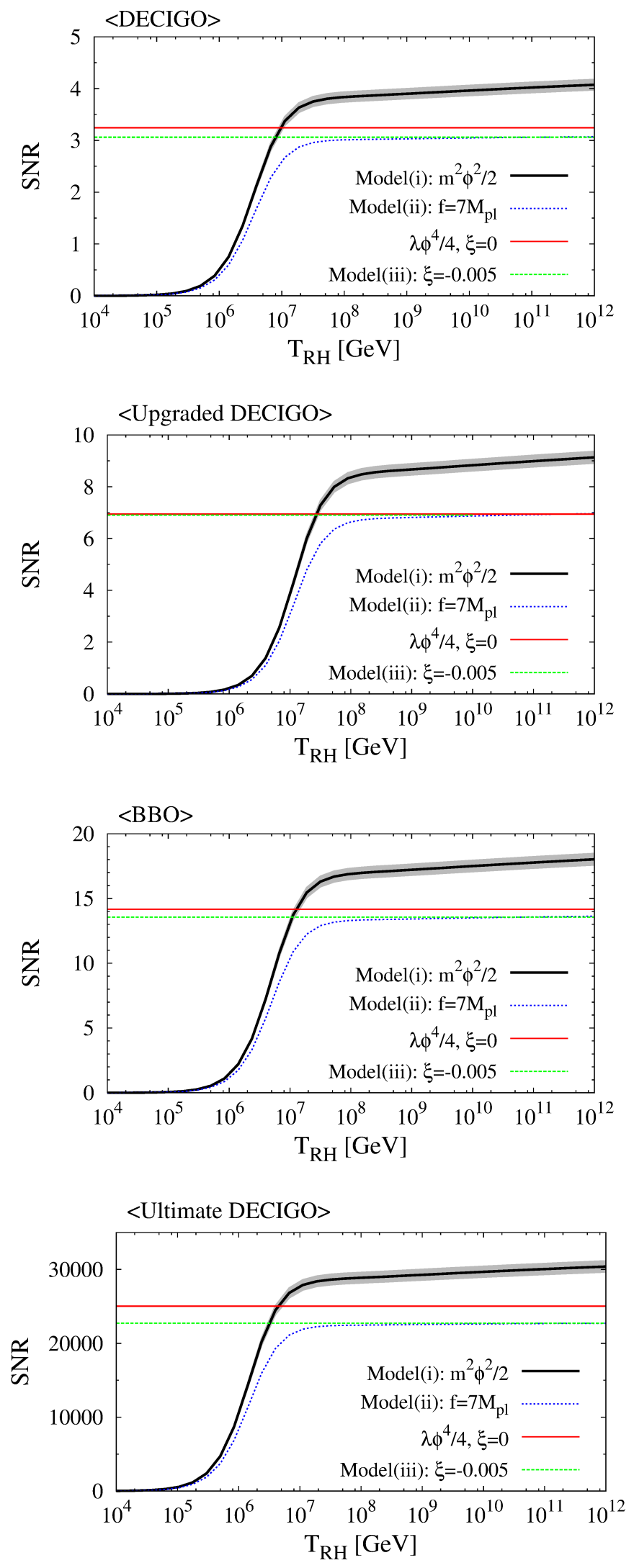

Figure 4: The SNR versus the reheating temperature computed for the model (i), the model (ii) with $f=7 M_{\mathrm{pl}}$, the potential $V(\phi)=\lambda \phi^{4} / 4$ with $\xi=0$, and the model (iii) with $\xi=-5.0 \times 10^{-3}$. Each panel corresponds to the SNR for DECIGO, upgraded DECIGO, BBO, and Ultimate DECIGO, from top to bottom, respectively. The gray region shows the $1 \sigma$ uncertainty in the amplitude of primordial curvature perturbations $\mathcal{P}_{\mathcal{R}}^{\text {prim }}=\left(2.200_{-0.054}^{+0.056}\right) \times 10^{-9}$ constrained from Planck [6]. For observation time of the gravitational wave, we assume $T_{\text {obs }}=10$ year. The SNR scales as $\propto \sqrt{T_{\text {obs }} / 10 \text { year. }}$ 

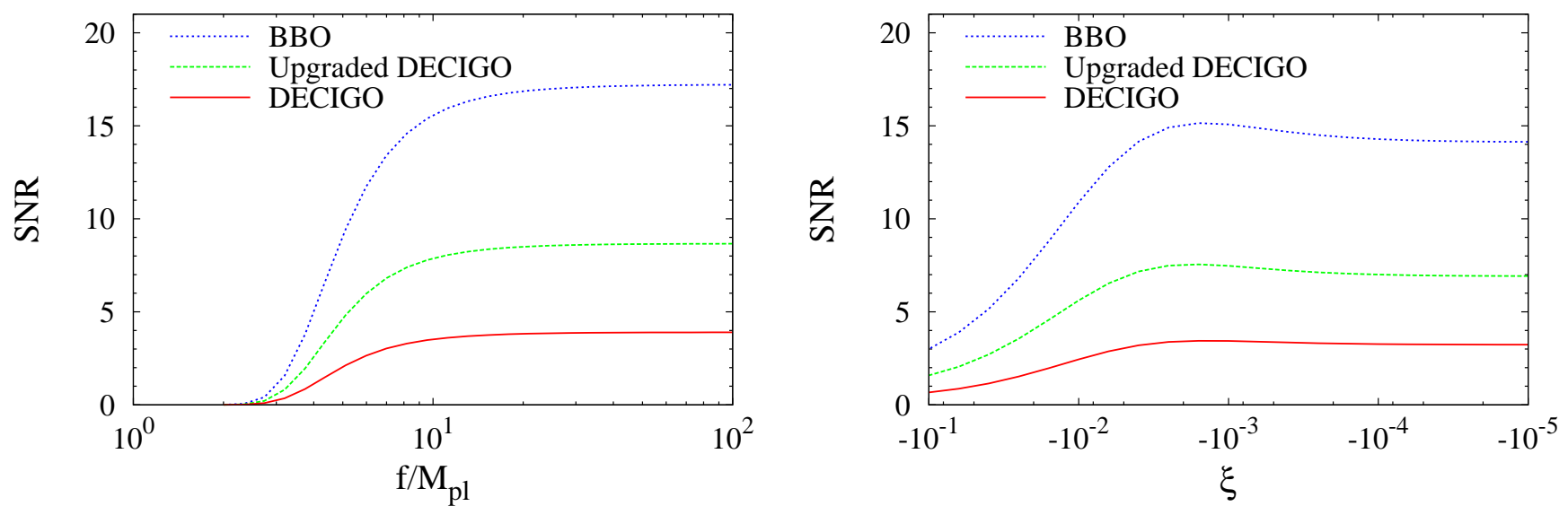

Figure 5: (Left) Dependence of the SNR on the parameter $f / M_{\mathrm{pl}}$ for the model (ii). (Right) Dependence of the SNR on the non-minimal coupling $\xi$ for the model (iii). Each line is the SNR calculated with noise curves of DECIGO, upgraded DECIGO and $\mathrm{BBO}$, respectively, with $T_{\mathrm{obs}}=10$ year. In both cases, we assume $T_{\mathrm{RH}}=10^{9} \mathrm{GeV}$.

If the B-mode polarization measurements convincingly confirm the existence of primordial gravitational waves with $r$ larger than 0.05, its direct detection by upgraded DECIGO and BBO could be also possible in the future.

\section{SUMMARY}

In this paper, we have discussed the possibility of the direct measurement of primordial gravitational waves by next generation interferometric detectors. The prospects for the direct detection depend on the ratio $r$ between tensor and scalar perturbations generated during inflation. If the B-mode polarization measurements of the CMB really confirm the presence of primordial tensor modes with $r$ of the order of 0.1 , it is expected that the interferometric detectors like DECIGO and BBO will be able to detect inflationary gravitational waves in the future.

We have focused on the three large-field inflationary models: quadratic chaotic, natural, and quartic chaotic with non-minimal couplings. The tensor-to-scalar ratio in the model (i) is larger than that in the model (ii) for a given number of e-foldings during inflation. We have studied the background dynamics after inflation including the discussion of the inflaton decay associated with the reheating temperature. The model (iii) with $|\xi| \ll 1$ can be distinguished from the models (i) and (ii), in that the reheating dynamics in the former is driven by a massless inflaton field rather than the massive field. This difference affects the resulting spectrum of the gravitational wave background. We have also precisely estimated the number of e-foldings relevant to the CMB anisotropies, which is important to place accurate constraints on inflationary models from the CMB.

We illustrated theoretical predictions (Sec. III) for the CMB observables in each inflationary model as well as the likelihood contours constrained from the joint data analysis of Planck $+\mathrm{WP}+\mathrm{BAO}+$ high- $\ell$ without employing the slow-roll approximation. Adding the recent BICEP2 data in the analysis leads to the bound: $0.07<r<0.25(95 \%$ C.L.). Since there is a tension between the Planck and BICEP2 data, we have not literally used the constraints derived from the BICEP2 data. The future independent B-mode polarization measurements will provide us with more convincing bounds on $r$.

We have computed the spectra of the gravitational wave background $\Omega_{\mathrm{GW}}$ for the parameter space inside the $95 \%$ C.L. boundary constrained by Planck+WP+BAO+high- $\ell$. For the models (i) and (ii) there is a suppression of $\Omega_{\mathrm{GW}}$ for the frequencies satisfying (54). In this case, the critical frequency $f_{\mathrm{RH}}$ depends on the reheating temperature $T_{\mathrm{RH}}$. Provided that $T_{\mathrm{RH}}$ is larger than the order of $10^{6} \mathrm{GeV}$, the models (i) and (ii) can reach the detection sensitivity of gravitational waves by DECIGO and BBO for $0.1 \mathrm{~Hz}<f<1 \mathrm{~Hz}$ (see Fig. 4). When $|\xi| \ll 1$, the model (iii) is not plagued by the sharp suppression of $\Omega_{\mathrm{GW}}$ due to the absence of a temporal matter era after inflation, so the direct detection is possible regardless of the value of $T_{\mathrm{RH}}$.

We have calculated the signal-to-noise ratio of DECIGO, upgraded DECIGO, BBO, and Ultimate DECIGO for the models (i)-(iii). Compared to the SNR of DECIGO computed in Ref. [41], we considered the upgraded version of DECIGO that improves the sensitivity of measurements. For the model (i) the direct detections by upgraded DECIGO and BBO are possible for $T_{\mathrm{RH}}>1.5 \times 10^{7} \mathrm{GeV}, T_{\mathrm{RH}}>2.8 \times 10^{6} \mathrm{GeV}$, and $T_{\mathrm{RH}}>4.0 \times 10^{3} \mathrm{GeV}$ at $5 \sigma$, 
respectively. For the model (ii) the upgraded DECIGO and BBO could detect the primordial gravitational waves at $5 \sigma$ for $f>5.3 M_{\mathrm{pl}}$ and $f>4.0 \mathrm{M}_{\mathrm{pl}}$, respectively, provided that $T_{\mathrm{RH}} \gtrsim 10^{8} \mathrm{GeV}$. For the model (iii) the direct detections would be feasible for $\xi>-0.013$ (upgraded DECIGO) and $\xi>-0.043$ (BBO).

We have thus shown that the large-field inflationary models with $r$ larger than the order of 0.01 leave interesting observational signatures in the gravitational wave background. In particular, the models in which reheating is driven by an effective massless inflaton field can be distinguished from the massive models with a temporal matter era after inflation. We hope that, in addition to the indirect detection of tensor perturbations from the B-mode polarization, the measurements by interferometric detectors will shed new light on the nature of primordial gravitational waves in the foreseeable future.

\section{ACKNOWLEDGEMENTS}

This work is supported by the Grant-in-Aid for Scientific Research from JSPS (Nos. 24540287 (TC), 24540286 (ST), 25287057(NS)), by the Grant-in-Aid for Scientific Research on Innovative Areas (No. 21111006 (ST)) and in part by Nihon University (TC) and by World Premier International Research Center Initiative (WPI Initiative), MEXT, Japan. Compared to the version published in Physical Review D, we also incorporated the sensitivity curves and the signal-to-noise ratios of Ultimate DECIGO in this updated arXiv version. We thank Takashi Nakamura for his suggestion to include the discussion of Ultimate DECIGO.

[1] A. A. Starobinsky, Phys. Lett. B 91, 99 (1980); K. Sato, Mon. Not. Roy. Astron. Soc. 195, 467 (1981); D. Kazanas, Astrophys. J. 241, L59 (1980); A. H. Guth, Phys. Rev. D 23, 347 (1981).

[2] V. F. Mukhanov and G. V. Chibisov, JETP Lett. 33, 532 (1981) [Pisma Zh. Eksp. Teor. Fiz. 33, 549 (1981)]; A. D. Linde, Phys. Lett. B 116, 335 (1982); S. W. Hawking, Phys. Lett. B 115, 295 (1982); A. A. Starobinsky, Phys. Lett. B 117, 175 (1982); A. H. Guth and S. Y. Pi, Phys. Rev. Lett. 49, 1110 (1982).

[3] A. A. Starobinsky, JETP Lett. 30, 682 (1979) [Pisma Zh. Eksp. Teor. Fiz. 30, 719 (1979)]; V. A. Rubakov, M. V. Sazhin and A. V. Veryaskin, Phys. Lett. B 115, 189 (1982); L. F. Abbott and M. B. Wise, Nucl. Phys. B 244, 541 (1984).

[4] G. F. Smoot et al., Astrophys. J. 396, L1 (1992); C. L. Bennett et al., Astrophys. J. 464, L1 (1996) astro-ph/9601067.

[5] C. L. Bennett et al. [WMAP Collaboration], Astrophys. J. Suppl. 148, 1 (2003) astro-ph/0302207]; D. N. Spergel et al. [WMAP Collaboration], Astrophys. J. Suppl. 148, 175 (2003) astro-ph/0302209].

[6] P. A. R. Ade et al. [Planck Collaboration], arXiv:1303.5076] [astro-ph.CO].

[7] J. E. Lidsey et al., Rev. Mod. Phys. 69, 373 (1997) astro-ph/9508078]; D. H. Lyth and A. Riotto, Phys. Rept. 314, 1 (1999) hep-ph/9807278; B. A. Bassett, S. Tsujikawa and D. Wands, Rev. Mod. Phys. 78, 537 (2006) astro-ph/0507632; D. Baumann, arXiv:0907.5424 [hep-th].

[8] P. A. R. Ade et al. [Planck Collaboration], arXiv:1303.5082 [astro-ph.CO].

[9] J. Martin, C. Ringeval and V. Vennin, arXiv:1303.3787] [astro-ph.CO].

[10] S. Tsujikawa, J. Ohashi, S. Kuroyanagi and A. De Felice, Phys. Rev. D 88, 023529 (2013) arXiv:1305.3044 [astro-ph.CO]]; S. Tsujikawa, PTEP 2014, no. 6, 06 B104 (2014) arXiv:1401.4688 [astro-ph.CO]].

[11] A. R. Liddle and D. H. Lyth, Phys. Lett. B 291, 391 (1992) arXiv:astro-ph/9208007.

[12] P. A. R. Ade et al. [BICEP2 Collaboration], arXiv:1403.3985 [astro-ph.CO].

[13] G. Hinshaw et al. [WMAP Collaboration], Astrophys. J. Suppl. 208, 19 (2013) arXiv:1212.5226 [astro-ph.CO]].

[14] M. J. Mortonson and U. Seljak, arXiv:1405.5857 [astro-ph.CO]; R. Flauger, J. C. Hill and D. N. Spergel, arXiv:1405.7351 [astro-ph.CO].

[15] M. Kamionkowski, A. Kosowsky and A. Stebbins, Phys. Rev. Lett. 78, 2058 (1997) astro-ph/9609132; U. Seljak and M. Zaldarriaga, Phys. Rev. Lett. 78, 2054 (1997) astro-ph/9609169.

[16] A. G. Polnarev, Sov. Ast., 29, 607, 1985.

[17] J. Lizarraga, J. Urrestilla, D. Daverio, M. Hindmarsh, M. Kunz and A. R. Liddle, Phys. Rev. Lett. 112, 171301 (2014) arXiv:1403.4924 [astro-ph.CO]]; A. Moss and L. Pogosian, Phys. Rev. Lett. 112, 171302 (2014) arXiv:1403.6105 [astroph.CO]].

[18] D. H. Lyth, Phys. Rev. Lett. 78, 1861 (1997) hep-ph/9606387; L. Boubekeur and D. H. Lyth, JCAP 0507, 010 (2005) hep-ph/0502047.

[19] A. D. Linde, Phys. Lett. B 129, 177 (1983).

[20] K. Freese, J. A. Frieman and A. V. Olinto, Phys. Rev. Lett. 65, 3233 (1990).

[21] T. Futamase and K. -i. Maeda, Phys. Rev. D 39, 399 (1989).

[22] R. Fakir and W. G. Unruh, Phys. Rev. D 41, 1783 (1990).

[23] F. L. Bezrukov and M. Shaposhnikov, Phys. Lett. B 659, 703 (2008) arXiv:0710.3755 [hep-th]].

[24] N. Makino and M. Sasaki, Prog. Theor. Phys. 86, 103 (1991); R. Fakir, S. Habib and W. Unruh, Astrophys. J. 394, 396 (1992); D. I. Kaiser, Phys. Rev. D 52, 4295 (1995) astro-ph/9408044; J. C. Hwang and H. Noh, Phys. Rev. Lett. 81, 
5274 (1998).

[25] E. Komatsu and T. Futamase, Phys. Rev. D 58, 023004 (1998) astro-ph/9711340; E. Komatsu and T. Futamase, Phys. Rev. D 59, 064029 (1999) astro-ph/9901127]; S. Tsujikawa and B. Gumjudpai, Phys. Rev. D 69, 123523 (2004) astro-ph/0402185; T. Chiba and M. Yamaguchi, JCAP 0810, 021 (2008) arXiv:0807.4965 [astro-ph]]; N. Okada, M. U. Rehman and Q. Shafi, Phys. Rev. D 82, 043502 (2010) arXiv:1005.5161 [hep-ph]]; A. Linde, M. Noorbala and A. Westphal, JCAP 1103, 013 (2011) arXiv:1101.2652 [hep-th]]; N. Okada, V. N. Senoguz and Q. Shafi, arXiv:1403.6403 [hep-ph].

[26] K. Kamada, T. Kobayashi, M. Yamaguchi and J. Yokoyama, Phys. Rev. D 83, 083515 (2011) arXiv:1012.4238 [astroph.CO]]; L. A. Popa, JCAP 1110, 025 (2011) arXiv:1107.3436 [astro-ph.CO]]; J. Ohashi and S. Tsujikawa, JCAP 1210, 035 (2012) arXiv:1207.4879 [gr-qc]].

[27] L. Amendola, Phys. Lett. B 301, 175 (1993) gr-qc/9302010; C. Germani and A. Kehagias, Phys. Rev. Lett. 105, 011302 (2010) arXiv:1003.2635 [hep-ph]]; C. Germani and Y. Watanabe, JCAP 1107, 031 (2011); S. Tsujikawa, Phys. Rev. D 85, 083518 (2012) arXiv:1201.5926 [astro-ph.CO]].

[28] N. Seto, S. Kawamura and T. Nakamura, Phys. Rev. Lett. 87, 221103 (2001) astro-ph/0108011; S. Kawamura et al., Class. Quant. Grav. 23, S125 (2006); S. Kawamura et al., Class. Quant. Grav. 28, 094011 (2011).

[29] S. Phinney et al., The big bang observer: direct detection of gravitational waves from the birth of the Universe to the present, NASA Mission Concept Study.

[30] M. S. Turner, Phys. Rev. D 55, 435 (1997) astro-ph/9607066].

[31] A. Cooray, Mod. Phys. Lett. A 20, 2503 (2005) astro-ph/0503118].

[32] T. L. Smith, M. Kamionkowski and A. Cooray, Phys. Rev. D 73, 023504 (2006) astro-ph/0506422.

[33] T. L. Smith, H. V. Peiris and A. Cooray, Phys. Rev. D 73, 123503 (2006) astro-ph/0602137.

[34] T. L. Smith, M. Kamionkowski and A. Cooray, Phys. Rev. D 78, 083525 (2008) arXiv:0802.1530 [astro-ph]].

[35] S. Chongchitnan and G. Efstathiou, Phys. Rev. D 73, 083511 (2006) astro-ph/0602594.

[36] B. C. Friedman, A. Cooray and A. Melchiorri, Phys. Rev. D 74, 123509 (2006) astro-ph/0610220.

[37] Y. Watanabe and E. Komatsu, Phys. Rev. D 73, 123515 (2006) astro-ph/0604176.

[38] T. Chiba, Y. Himemoto, M. Yamaguchi and J. Yokoyama, Phys. Rev. D 76, 043516 (2007) arXiv:0705.2851 [gr-qc]].

[39] N. Seto and J. Yokoyama, J. Phys. Soc. Jap. 72, 3082 (2003) gr-qc/0305096;

[40] K. Nakayama, S. Saito, Y. Suwa, and J. Yokoyama, Phys. Rev. D77, 124001 (2008). arXiv:0802.2452 [hep-ph]]; K. Nakayama, S. Saito, Y. Suwa, and J. Yokoyama, JCAP 0806, 020 (2008) arXiv:0804.1827 [astro-ph]].

[41] S. Kuroyanagi, T. Chiba and N. Sugiyama, Phys. Rev. D 83, 043514 (2011) arXiv:1010.5246 [astro-ph.CO]].

[42] A. Albrecht, P. J. Steinhardt, M. S. Turner and F. Wilczek, Phys. Rev. Lett. 48, 1437 (1982).

[43] A. D. Dolgov and A. D. Linde, Phys. Lett. B 116, 329 (1982); L. F. Abbott, E. Farhi and M. B. Wise, Phys. Lett. B 117, $29(1982)$.

[44] L. Kofman, A. D. Linde and A. A. Starobinsky, Phys. Rev. D 56, 3258 (1997) hep-ph/9704452.

[45] E. W. Kolb and M S. Turner, "The Early Universe" (Westview Press Boulder, CO, 1990).

[46] L. Dai, M. Kamionkowski and J. Wang, arXiv:1404.6704 [astro-ph.CO].

[47] J. M. Bardeen, Phys. Rev. D 22, 1882 (1980).

[48] K. -i. Maeda, Phys. Rev. D 39, 3159 (1989).

[49] R. Catena, M. Pietroni and L. Scarabello, Phys. Rev. D 76, 084039 (2007) astro-ph/0604492; N. Deruelle and M. Sasaki, Springer Proc. Phys. 137, 247 (2011) arXiv:1007.3563 [gr-qc]]; T. Chiba and M. Yamaguchi, JCAP 1310, 040 (2013) arXiv:1308.1142 [gr-qc]].

[50] http://cosmologist.info/cosmomc/

[51] A. Lewis, Phys. Rev. D87, 103529 (2013) arXiv:1304.4473 [astro-ph.CO]].

[52] F. Beutler et al., Mon. Not. Roy. Astron. Soc. 416, 3017 (2011) arXiv:1106.3366 [astro-ph.CO]]; N. Padmanabhan et al., arXiv:1202.0090 [astro-ph.CO]; L. Anderson et al., Mon. Not. Roy. Astron. Soc. 427, no. 4, 3435 (2013) arXiv:1203.6594 [astro-ph.CO]].

[53] S. Das et al., arXiv:1301.1037 [astro-ph.CO]; C. L. Reichardt et al., Astrophys. J. 755, 70 (2012) [arXiv:1111.0932 [astroph.CO]].

[54] A. A. Starobinsky, Phys. Lett. B 91, 99 (1980).

[55] S. Kuroyanagi, T. Chiba and N. Sugiyama, Phys. Rev. D 79, 103501 (2009) arXiv:0804.3249 [astro-ph]].

[56] M. Kamionkowski, A. Kosowsky and M. S. Turner, Phys. Rev. D 49, 2837 (1994) arXiv:astro-ph/9310044.

[57] S. Weinberg, Phys. Rev. D 69, 023503 (2004) astro-ph/0306304.

[58] B. Allen and J. D. Romano, Phys. Rev. D 59, 102001 (1999) arXiv:gr-qc/9710117.

[59] H. Kudoh, A. Taruya, T. Hiramatsu and Y. Himemoto, Phys. Rev. D 73, 064006 (2006) arXiv:gr-qc/0511145.

[60] V. Corbin and N. J. Cornish, Class. Quant. Grav. 23, 2435 (2006) gr-qc/0512039.

[61] K. Yagi and N. Seto, Phys. Rev. D 83, 044011 (2011) arXiv:1101.3940 [astro-ph.CO]].

[62] T. A. Prince, M. Tinto, S. L. Larson and J. W. Armstrong, Phys. Rev. D 66, 122002 (2002) arXiv:gr-qc/0209039. 